\title{
The Ethical Unjustifications of COVID-19 Triage Committees
}

\author{
Yi Jiao (Angelina) Tian
}

Received: 4 January 2021 / Accepted: 5 July 2021 / Published online: 29 December 2021

(C) Journal of Bioethical Inquiry Pty Ltd. 2021

\begin{abstract}
The ever-debated question of triage and allocating the life-saving ventilator during the COVID-19 pandemic has been repeatedly raised and challenged within the ethical community after shortages propelled doctors before life and death decisions (Anderson-Shaw and Zar 2020; Huxtable 2020; Jongepier 2020; Peterson, Largent, and Karlawish 2020). The British Medical Association's ethical guidance highlighted the possibility of an initial surge of patients that would outstrip the health system's ability to deliver care "to existing standards," where utilitarian measures have to be applied, and triage decisions need to maximize "overall benefit" (British Medical Association 2020, 3) In these emergency circumstances, triage that "grades according to their needs and the probable outcomes of intervention" will prioritize or eliminate patients for treatment, and health professionals may be faced with obligations to withhold or withdraw treatments to some patients in favour of others (British Medical Association 2020, 4). This piece is a response and extension to articles published on the manner of involvement for ethics and ethicists in pandemic triage decisions, particularly examining the ability and necessity of establishing triage committees to ameliorate scarce allocation decisions for physicians.
\end{abstract}

Y. J. (A.) Tian ( $\varangle)$

Institute for Biomedical Ethics, University of Basel,

Bernouillistrasse 28, CH-4056 Basel, Switzerland

e-mail: angelina.tian@unibas.ch
Keywords Ventilator triage - Triage Committees · COVID-19 · Moral distress · Procedural ethical guidance

\section{Background}

The decision to allocate or withdraw life-saving medical treatments during resource scarcity and patient surge is neither a pandemic-specific nor novel challenge in bioethics (Persad, Wertheimer, and Emanuel 2009; McGough et al. 2005; Becker 1979; Aulisio 2016; Burkle 2006; Devereaux et al. 2008). During the COVID-19 pandemic, many severely ill patients relied on mechanical ventilators to sustain their breathing (Truog et al. 2020). The scarcity of medical equipment supply to meet swelling demand forced providers to triage and prioritize patients, rationing in a similar manner to the distribution of antiretroviral therapies in developing countries or the allocation of kidney dialysis in 1960s Seattle (Persad, Wertheimer, and Emanuel 2009; McGough et al. 2005; Aulisio 2016). As the patient's breathing deteriorates below self-sustaining levels, a ventilator may be the sole factor in determining whether she can prolong survival in time for further treatments (Liddell et al. 2020). As such, there is particular significance in focusing ethical attention on ventilator allocation in the COVID19 pandemic.

In the context of medical surges, when the number of patients who require ventilation support increases, 
a temporary and rapid increase of ventilators or beds may reduce the need to engage with the thorny questions of allocation (Huxtable 2020; Chuang et al. 2020). However, this only shifts the focus of allocation and prioritization of scarce medical resources, as the availability of staff is still limited in the shortterm (Huxtable 2020; Chuang et al. 2020). Therefore, this paper will employ the term "ventilator triage" to refer to all resource allocation necessary for treating a critically ill COVID-19 patient, including beds or medical staff availability, as providers' ability to receive and treat COVID-19 patients depends on more than ventilator supply (Feinstein et al. 2020; Matheny Antommaria et al. 2020).

The overall discussion of triage contextualized in the COVID-19 pandemic can be streamlined to four major debates: the need for triage criteria versus relying only on clinical judgement; the types of considerations upon which the triage criteria is established, which are currently heterogeneous amongst hospitals with triage protocols that vary between the "utilitarian ... egalitarian ... contractualism ... flattening the curve ...[and] paternalism approach[es]"; whether to prioritize healthcare workers in COVID-19 treatment; and finally, the need for a separate triage committee that isolates allocation decisions to an independent group of physicians, laypeople, and administrators (Emanuel et al. 2020; Savulescu and Wilkinson 2020; Truog, Mitchell, and Daley 2020; White and Lo 2020; Feinstein et al. 2020; Huxtable 2020; Camporesi and Mori 2020; Sprung et al. 2020).

This paper will only address the last debate, taking the position to justify the harms and inefficiencies in establishing a triage committee distinct from bedside clinicians. In other words, the present argument grapples specifically with the procedural question that arises from triaging COVID-19 patients: who should be in charge of making treatment decisions in a pandemic (Huxtable 2020; Camporesi and Mori 2020)?

\section{Ethically Unjustifying the Gold Standard}

While the emerging consensus or, "gold standard," for the ethics of triage is to create separate teams whose decisions on treatment allocation are independent from clinical staff, this paper argues that triage decisions should ultimately be made by clinicians who are guided by clear, regularly updated triage criteria that is adapted to resource availability (Feinstein et al. 2020). While these independent triage committees are common strategies which U.S. hospitals employ to prepare for the pandemic, this trend has not been widely adopted in some European countries (Orfali 2020). Recommendations in some European countries and from some ethicists call for triaging and ventilator allocation under the clinician's jurisdiction, guided solely by clinical judgement (Orfali 2020; Vergano et al. 2020). Ethics literature has outlined the intentions behind separating clinicians from triage decisions, which include minimizing moral distress, increasing objectivity and consistency, avoiding conflicts of goals, and increasing efficiency (Truog, Mitchell, and Daley 2020; Rosenbaum 2020; Feinstein et al. 2020; Dunham, Rieder, and Hymbryd 2020; Emanuel et al. 2020; Robert et al. 2020). Given these theoretical and geographical differences in the ethical community, this paper will list three reasons that have been put forward in support of triage committees, detailing the arguments in favour of each and point out their possible flaws, and then make with a few recommendations for future discussions.

\section{Ethical Unjustification I: Moral Distress}

Medical resource shortage reports from hospitals in Italy and China described physicians in debilitating moral distress and angst while facing the decisions allocating ventilators to critically ill patients (Truog, Mitchell, and Daley 2020; Rosenbaum 2020; Camporesi and Mori 2020; Huxtable 2020). In Huxtable's articles, he emphasizes the initial vacuum and therefore necessity of ethical guidance for healthcare professional facing this distress (Huxtable 2020; Fritz et al. 2020). To buffer clinicians from this potential emotional harm and distribute the moral distress from a single individual, ethicists argued for the establishment of separate triage committees to take the responsibility from clinicians (Truog, Mitchell, and Daley 2020; White and Lo 2020; Feinstein 2020). This approach allows frontline clinicians to maintain their "traditional roles as fiduciary advocates" and caretakers, while the committee can collectively ensure "consistent and unbiased decisions across patient groups" (Truog, Mitchell, and Daley 2020, 1974). Ethicists recommend that the triage committee create and update rationing criteria, make allocation 
decisions, communicate the decisions to the families, and support physicians in the process of withdrawing ventilation (Truog, Mitchell, and Daley 2020). The creation of a triage committee would theoretically eliminate moral distress and ensure clear communication between clinicians, patients, and their families (Truog, Mitchell, and Daley 2020).

Other ethicists also believe in the face of morally challenging scenarios, it is best to separate those clinicians who provide care from those making triage decisions, where decisions can then be reviewed by another "centralized state-level monitoring committee" for appeals and inequities (Rosenbaum 2020, 1875; Feinstein et al., 2020). If clinicians are required to consider resource allocation when facing individual patients, they betray values inherent to their professional duties, resulting in conflicts of interest and moral distress (Dunham, Rieder, and Hymbryd 2020; Sheahan and Lamont 2020). Thereby, an independent team should review patient profiles and make decisions at the collective level that do not conflict with clinical ethics that frontline staff are also bound by (Dunham, Rieder, and Hymbryd 2020).

Despite these arguments, empirical data supporting the claim that moral distress may be minimized with separate triage officers or team is lacking (Zivot 2020). It is important here to define that moral distress can arise when "external or internal constraints preclude the performance of an ethically appropriate choice or action" (Dunham, Rieder, and Humbyrd 2020, 4). If establishing separate triage committees rests upon the justification of reducing moral distress, it may depend therefore on whether physicians perceive that the triage decisions made are appropriate or ethically justified. If scenarios arise where clinicians witness decisions incongruent with their own moral principles, they may experience moral residue that makes future decisions more intolerable, ultimately leading to moral injury (Dunham, Rieder, and Humbyrd 2020).

An empirical bioethics piece by Chuang et al. (2020) analysed the potential barriers, emotional and practical, in the implementation of triage protocols during and in anticipation COVID-19 ventilator shortages. They found that establishing a separate triage team and relieving clinicians of their decision-making ability may theoretically reduce their burdens, but there are concerns that this protocol may "represent a threat to the participants' sense of self" and "threaten the fiduciary duty of healthcare providers to advocate for their individual patients" (Chuang et al. 2020, 153). Furthermore, while clinical ethics value both patient and physician autonomy in decision-making, a separate triage committee founded on public health ethics principles may "abdicate the physician's authority" and thereby their autonomy (Chuang et al. 2020, 153). One participant in the empirical research process mentioned the Milgram experiment to demonstrate the concerns for a blind obedience to authority and giving excess power to the triage committee (Chuang et al. 2020). In other words, even if clinicians do not need to make decisions to withhold or withdrawal life-sustaining treatments, they are not exempt from the necessity to act upon them. Therefore, triage committees may end up taking away physicians' sense of authority and distorting their self-image as medical professionals in addition to inflicting frustration and moral conflict on physicians when decisions made do not reflect their clinical judgement.

The allocation and particularly reallocation of medical resources in order to maximize benefit for all will "require a departure from the usual fiduciary duty of the bedside clinicians," changing physicians' traditional roles and identities from patient advocates to the implementer of crisis-level triage standards (Chuang et al. 2020, 148). The shift in obligations may generate clinician discomfort, especially during ventilator withdrawal, as it runs counter to the clinician's duty to the patient and the commitment to clinical ethics principles of maleficence and beneficence (O'Laughlin and Hick 2008; Chuang et al. 2020). In other words, when clinicians refuse to treat patients "on the basis of [their] claim of moral distress," this action may be deemed inherently maleficent and against their doctrine in clinical ethics (Zivot 2020, 1242). Although moral distress can be ameliorated with triage guidelines in emergency and confusing situations, guidelines or decisions that are ethically unacceptable to physicians may result in worsened moral distress (Chuang et al. 2020). If clinicians perceive the triage decisions to be invalid or have concerns regarding legal repercussions, moral conflict will not ease with the establishment of triage committees. 


\section{Ethical Unjustification II: Public Health Ethics vs. Clinical Ethics}

The next ethical unjustification of triage committees is relevant to the shift from clinical to public health ethics during the allocation of scarce resources and epidemics (Royo-Bordonada and Roman-Maestre 2015; O'Neill 2002). Due to the traditional role of clinicians as individual patient-advocates, some ethicists suggest the delegation of all triage decisions to a distinct decision-making committee to prevent clinicians from needing to move away from their clinical ethics principles, as priorities shift from benefit and maximizing population health (Feinstein et al. 2020; Dunhan, Rieder, and Humbyrd 2020; White and Lo 2020; Sheahan and Lamont 2020). However, because triage protocols based in public health ethics evaluate on a population level, this may result in category exclusions that may also be inherently biased and discriminatory on the individual level (White and Lo 2020; Savin and Guidry-Grimes 2020; Italian National Bioethics Committee Opinion 2020). Therefore, this section argues that, in comparison with triage committees with members who operate potentially without clinical expertise and first-hand information from patients, the harm from category exclusions during triage may be mediated if decisions remain with frontline clinicians.

Ethicists argue that in public health emergencies, the health benefits of the population may override the ethical obligations of the physician to individual patients (White and Lo 2020). The goals of public health ethics necessarily diverge from those of clinical practice and take into consideration ethical conflicts that arise when the health needs of the community are at stake, such as the allocation of scarce resources (Royo-Bordonada and Roman-Maestre 2015; Pagel 2020). Clinical ethics are bound in principle by voluntary relationships between patients and physicians that respect the autonomies of both parties and the patients' just access to healthcare, where "compulsion is unacceptable outside narrowly defined areas" (O'Neill 2002, 39). However, public health ethics cannot rule out the possibility that actions implemented by governments may negatively impact some individuals at the expense of others (Royo-Bordonada and Roman-Maestre 2015). Specifically in the process of developing rationing criteria for COVID19 , triage policies may consider various population characteristics that may categorically exclude patients of a certain medical condition or age status (White and Lo 2020; Savin and Guidry-Grimes 2020). For example, as triage criteria become shared for public discussion during the COVID-19 pandemic, disability advocates argued that these protocols risked devaluing the disabled as a population, as well as those with pre-existing conditions or mentally challenged people, fearing that such triage criteria exacerbates existing ableism in healthcare (Savin and Guidry-Grimes 2020).

Ethicists who recommend triage committees often value the committees' contributions in responsibilities, perspectives, and experiences that differ from clinicians, in addition to their physical and moral distances from patients in question (Truog, Mitchell, and Daley 2020; Feinstein 2020; Kuschner, Pollard, and Ezeji-Okoye 2007). A qualitative study on ventilator triage policy research in U.S. hospitals found that half of the analysed policies required or recommended members of the triage team not be involved in direct patient care (Matheny Antommaria et al. 2020). The policies also recommended that the triage team's composition be varied to include chaplains and ethicists, as well as clinicians and nurses (Matheny Antommaria et al. 2020). However, there is potential for bias when all aspects of an individual patient's situation need to be relayed from the bedside. This study also found that policies which "rely on triage teams ... may introduce implicit bias or discrimination" (Matheny Antommaria et al. 2020, 5). In addition, as few policies in this study specify "blinding mechanisms" to prevent triage teams from accessing information such as race or the ability to pay, it is indeed unclear that separate allocation teams will not contribute to inequities in care (Matheny Antommaria et al. 2020, 5).

As population health indicators are prioritized during triage, this paper goes on to argue that the reliance of triage officers distanced from frontlines may be unproductive, particularly when non-clinicians are included with less knowledge of clinical expertise. Although it is certainly arrogant to suggest that decisions from frontline clinicians will always be free of bias, especially in times of resource scarcity and under triage guidance, their distance to the frontlines and access to clinical judgement may provide more certainty that immediate and medically relevant information is considered during triage. Furthermore, as 
the allocation decisions made by triage committees are dependent on the composition of its members, fairness and equity are only possible if the committee is composed of individuals to form a "workable, moral, and lawful" triage system replicable across various hospitals that replaces an otherwise questionably biased group of clinicians (Zivot 2020, 1241). Therefore, while triage committees are valued for their differing perspectives, they may not necessarily ensure greater certainty against discriminating individual patients when public health ethics prioritizes population health.

To conclude this section, this paper builds upon flaws presented by separating triage from bedside, thereby suggesting the benefits of reverting back to clinical judgement for individual ventilator allocation decisions. The principles of public health ethics remain crucial in the development of guidelines for bedside clinicians, though they should not indicate the justification for a separate committee in this context. Furthermore, for a committee of individuals to judge each triage decision or for ethicists to agree on triage committee membership, significant time is required. This may result in delays and is unproductive for the dynamic and fast-moving environment that warranted triage in the first place.

\section{Ethical Unjustification III: Delay}

According to the empirical bioethics article by Chuang et al., some clinicians worry that the presence of a separate triage team may increase delays to the process of allocation, decreasing efficiency due to increased distance from the frontlines to the decisionmakers (Chuang et al. 2020). Although this distance may increase objectivity and decrease emotional distress in communicating triage decisions, any changes in the dynamic pandemic environment may result in frontline staff having to respond rapidly. In this situation, if physicians choose to override the triage team due to time-constraints, this decreases the efficacy of the presence of triage teams. However, if physicians choose to wait, this may forgo chances to maximize benefit for the population or their patients.

As most empirical data on triage teams and their effects on efficiency were collected prior to the pandemic, when triage teams are made up of frontline clinicians and nurses, there is not a convincing study on the comparison between triage decisions made by clinicians and non-frontline triage teams. Under nonpandemic circumstances, systematic reviews on triage teams composed of either a senior physician or a triage-liaison physician (TLP) to reduce delays provided varying results and study qualities, making it difficult to recommend a separate decision-making apparatus, even under direct leadership of a frontline physician (Centre for Reviews and Dissemination 2015; Rowe et al. 2011). Nevertheless, though the hypothesis that triage teams necessarily increase response times due to ventilator allocation is subsequently unproven, it also remains unclear that triage teams in this specific composition away from frontlines could result in efficiencies to solve crowding issues in COVID19. Therefore, while there is worry that independent teams may delay the decision-making process, there is also an immediate need to develop concrete empirical data for this link under the context of triaging ventilators for COVID-19 patients.

\section{Recommendations}

In the above sections, this paper argued against the establishment of triage committees and advocated for only frontline clinicians to make ventilator allocation decisions. However, it is crucial to note that clinicians should and can be supported in other ways by ethicists. Therefore, this section will list some recommendations for future triage decisions, which will take into account the problems listed in this paper, while being able to support clinicians adequately during distressing hospital settings in triage.

While public discussions on controversial issues should always be welcomed, there has been a wealth of ethicists' opinions that have not reached a clear conclusion for consistent policy implementation on triage committees. As triage is not a novel problem in medical ethics, recommendations resembling the fair-innings principle, egalitarian principles of the lottery or "first come, first served" approach, or the prioritization of certain population and professional groups have all been discussed and evaluated repeatedly (Persad, Wertheimer, and Emanuel 2009; Liddell et al. 2020; Savulescu and Wilkinson 2020). However, as ethicists struggle to understand their roles in this pandemic, their training to engage and 
welcome differences in opinion will result in controversial public debates (Jongepier 2020a, 2020b; Shaw 2020).

In the same way, ethical literature that justifies separate triage committees is well-intentioned and suggested by ethicists who want to participate and support frontline staff. However, there is also much controversy on the make-up of the triage committees. Would "only a senior physician with triage experience" have the authority or "can nurses, social workers and ethicists" also weigh in (Sprung et al. 2020, 1199). As such, these discussions around decisionmaking triage procedures can waste time and generate stress on the general public during an already stressful pandemic. While traditionally treatments are determined by clinicians through clinical judgement, patients are now unable to know exactly who will be determining their fates.

Although this paper recommended against separating triage decisions from bedside clinicians and addressed the drawbacks from an oversaturation of ethical discussions on triage, it is not dissuading ethicists from contributing to these issues during medical emergencies or pandemics. Bioethicists can provide other forms of guidance to clinicians while introducing the theoretical perspectives to applied ethical issues. Instead of joining an ad-hoc triage committee or evaluating previously discussed principles in public discussions, bioethicists' time and expertise can be more efficiently spent drafting and updating triage guidelines according to feedback from clinicians. In this way, clinicians are supported with theoretically backed and timely guidelines without being confronted with decisions contrary to their moral principles. Nor are they required to reiterate their frontline experiences to another committee and endure risks from decision-making delays. Clinicians are thus allowed to exercise their clinical judgement as patient-advocates within a structured decisionmaking apparatus during a pandemic. Although comprehensive and widely used, current instruments for triage decision-making such as SOFA and PELOD-2 have been questioned either for their simplicity or need for additional tests, demonstrating the need for additional development of triaging tools (dos Santos et al. 2020). Furthermore, as there are geographicaland cultural-dependent disparities in triage criteria, the presence of ethicists can provide timelier and situation-specific guidance to triage decisions.

\section{Conclusions}

This paper argued against establishing separate triage committees responsible for allocating life-saving ventilators during pandemics. There are disparities within the ethics community on the manner and extent of ethicist involvement in pandemic triage guidance, as well as the adoption differences that vary across countries and cultures (Orfali 2020; Ehni, Wiesing, and Ranisch 2020; Jongepier 2020). This paper engaged with the procedural aspect of "who" shall make the allocation decisions and argued against the establishment of triage committees. While clinical judgement should not be the only criteria for triage during the COVID-19 pandemic, the decision to allocate ventilators to certain patients over others should be reserved for the frontlines. Physicians should have control over their patients under critical conditions and maintain authority to make triage decisions guided by clear exclusion criteria. Although independent decision-making teams may reduce moral stress, help navigate changing ethical obligations, and increase democracy, triage committees can also introduce moral conflict and inefficiencies. Ethicists and established triage committees have many roles to play during the COVID-19 pandemic. Ultimately, both should work together to continuously inform and update triage guidelines for physicians facing debilitating and morally distressing decisions.

Acknowledgements The author was primarily supervised by Prof. Dr. Silvia Camporesi at the King's College London while this article was conceptualised and written.

\section{Declarations}

The author has nothing further to declare.

Competing Interests The author declares that there are no competing interests

\section{References}

Anderson-Shaw, L. and F. Zar. 2020. COVID-19, moral conflict, distress, and dying alone. Journal of Bioethical Inquiry 17(4): 777-782.

Aulisio, M. 2016. Why did hospital ethics committees emerge in the U.S.? AMA Journal of Ethics 18(5): 546-553.

Becker, E. 1979. Finite resources and medical triage. The American Journal of Medicine, 66(4): 549-550. 
British Medical Association. 2020. COVID-19-Ethical issues. A guidance note. United Kingdom: bma.uk.org (BMA).

Burkle, F. 2006. Population-based triage management in response to surge-capacity requirements during a largescale bioevent disaster. Academic Emergency Medicine 13(11): 1118-1129.

Camporesi, S., and M. Mori. 2020. Ethicists, doctors and triage decisions: Who should decide? And on what basis? Journal of Medical Ethics 0(1-3): https://doi.org/10.1136/ medethics-2020-106499.

Centre for Reviews and Dissemination. 2015. Triage and minimising crowding in emergency departments. Effectiveness Matters, January. www.york.ac.uk/crd/publications/effec tiveness-matters/triage-emergency-crowding/. Accessed September 8, 2021.

Chuang, E., P. Cuartas, T. Powell, and M. Gong. 2020. "We're not ready, but I don't think you're ever ready." Clinician perspectives on implementation of crisis standards of care. AJOB Empirical Bioethics 11(3):148-159.

Devereaux, A., J. Dichter, M. Christian,et al. 2008. Definitive care for the critically ill during a disaster: A framework for allocation of scarce resources in mass critical care. Chest 133(5): 51S-66S.

dos Santos, M., M. Martins, F. Santana, et al. 2020. COVID19: Instruments for the allocation of mechanical ventilators-a narrative review. Critical Care 24(1): 1-10.

Dunham, A., T. Rieder. and C. Humbyrd. 2020. A bioethical perspective for navigating moral dilemmas amidst the COVID-19 pandemic. Journal of the American Academy of Orthopaedic Surgeons 28(11): 471-476.

Ehni, H., U. Wiesing, and R. Ranisch. 2020. Saving the most lives-A comparison of European triage guidelines in the context of the COVID-19 pandemic. Bioethics 35(2): 125-134.

Emanuel, E., G. Persad, R. Upshur, et al. 2020. Fair allocation of scarce medical resources in the time of COVID-19. New England Journal of Medicine 382(21): 2049-2055.

Feinstein, M., J. Niforatos, I. Hyun, et al. 2020. Considerations for ventilator triage during the COVID-19 pandemic. The Lancet Respiratory Medicine. Epub ahead of print. https:// doi.org/10.1016/S2213-2600(20)30192-2.

Fritz, Z., R. Huxtable, J. Ives, A. Paton, A. Slowther, and D. Wilkinson 2020. Ethical road map through the COVID-19 pandemic. BMJ 369: $\mathrm{m} 2033$.

Huxtable, R. 2020. COVID-19: Where is the national ethical guidance? BMC Medical Ethics 21(1): 32.

Italian National Bioethics Committee Opinion. 2020. COVID19: Clinical decision-making in conditions of resource shortage and the "Pandemic Emergency Triage" criterion. Comitato Nazionale per la Bioetica.

Jongepier, F. 2020a. Ethicists, hold your horses (Part 1). Cardiff University Blogs: Open for Debate, May 18. https:// blogs.cardiff.ac.uk/openfordebate/2020/05/18/ethicistshold-your-horses-part-1/. Accessed September 8, 2021.

Jongepier, F. 2020b. Ethicists, hold your horses (Part 2). Cardiff University Blogs: Open for Debate, June 1. https:// blogs.cardiff.ac.uk/openfordebate/2020/06/01/ethicistshold-your-horses-part-2/. Accessed September 8, 2021.

Kuschner, W., J. Pollard, and S. Ezeji-Okoye. 2007. Ethical triage and scarce resource allocation during public health emergencies: Tenets and procedures. Hospital Topics 85(3): 16-25.

Liddell, K., J. Skopek, S. Palmer, S. Martin, J. Anderson, and A. Sagar. 2020. Who gets the ventilator? Important legal rights in a pandemic. Journal of Medical Ethics 46(7): 421-426.

Matheny Antommaria, A., T. Gibb, A. McGuire, et al. 2020. Ventilator triage policies during the COVID-19 pandemic at U.S. hospitals associated with members of the Association of Bioethics program directors. Annals of Internal Medicine 173(3): 188-194.

McGough, L., S. Reynolds, T. Quinn, and J. Zenilman. 2005. Which patients first? Setting priorities for antiretroviral therapy where resources are limited. American Journal of Public Health 95(7): 1173-1180.

Orfali, K. 2020. What triage issues reveal: Ethics in the COVID-19 pandemic in Italy and France. Journal of Bioethical Inquiry 17(4): 675-679.

O'Laughlin, D. and J. Hick. 2008. Ethical issues in resource triage. Respiratory Care 53(2): 190-200.

O'Neill, O. 2002. Public health or clinical ethics: Thinking beyond borders. Ethics \& International Affairs 16(2): 35-45.

Pagel, C. 2020. COVID-19: How to triage effectively in a pandemic [Blog]. The BMJ Opinion, March 9. https://blogs. bmj.com/bmj/2020/03/09/covid-19-triage-in-a-pandemicis-even-thornier-than-you-might-think/. Accessed September 9, 2021.

Persad, G., A. Wertheimer, and E. Emanuel. 2009. Principles for allocation of scarce medical interventions. The Lancet 373(9661): 423-431.

Peterson, A., E. Largent, and J. Karlawish. 2020. Ethics of reallocating ventilators in the COVID-19 pandemic. BMJ 369: 1828.

Rosenbaum, L. 2020. Facing COVID-19 in Italy-Ethics, logistics, and therapeutics on the epidemic's front line. New England Journal of Medicine 382(20): 1873-1875.

Robert, R., N. Kentish-Barnes, A. Boyer, A. Laurent, E. Azoulay, and J. Reignier. 2020. Ethical dilemmas due to the COVID-19 pandemic. Annals of Intensive Care 10(1): $1-9$.

Royo-Bordonada, M., and B. Román-Maestre. 2015. Towards public health ethics. Public Health Reviews 36(1): 1-15.

Savin, K., and L. Guidry-Grimes. 2020. Confronting disability discrimination during the pandemic. The Hastings Center, April 2. https://www.thehastingscenter.org/confrontingdisability-discrimination-during-the-pandemic/. Accessed September 9, 2021.

Savulescu, J., and D. Wilkinson. 2020. Who gets the ventilator in the coronavirus pandemic? These are the ethical approaches to allocating medical care. $A B C$ News, March 18. https://www.abc.net.au/news/2020-03-18/ethics-ofmedical-care-ventilator-in-the-coronavirus-pandemic/ 12063536. Accessed September 9, 2021.

Shaw, D. 2020. Triaging ethical issues during a pandemic: A rough guide [blog]. Journal of Medical Ethics Blog, June 22. https://blogs.bmj.com/medical-ethics/2020/06/22/triag ing-ethical-issues-during-a-pandemic-a-rough-guide/. Accessed September 9, 2021.

Sheahan, L., and S. Lamont. 2020. Understanding ethical and legal obligations in a pandemic: A taxonomy of "duty" for 
health practitioners. Journal of Bioethical Inquiry 17(4): 697-701.

Sprung, C., G. Joynt, M. Christian, R. Truog, J. Rello, and J. Nates. 2020. Adult ICU triage during the coronavirus disease 2019 pandemic. Critical Care Medicine, 48(8): 1196.

Truog, R., C. Mitchell, and G. Daley. 2020. The toughest triage-Allocating ventilators in a pandemic. New England Journal of Medicine 382(21): 1973-1975.

Rowe, B., X. Guo, C. Villa-Roe, et al. 2011. The role of triage liaison physicians on mitigating overcrowding in emergency departments: A systematic review. Academic Emergency Medicine 18(2): 111-120.

Vergano, M., G. Bertolini, A. Giannini, et al. 2020. Clinical ethics recommendations for the allocation of intensive care treatments in exceptional, resource-limited circumstances - Version N. 1. SIAARTI.

White, D., and B. Lo. 2020. A framework for rationing ventilators and critical care beds during the COVID-19 pandemic. JAMA 323(18): 1773.

Zivot, J. 2020. Coronavirus disease 2019 triage teams: Death by numbers*. Critical Care Medicine 48(8): 1241-1242.

Publisher's note Springer Nature remains neutral with regard to jurisdictional claims in published maps and institutional affiliations. 